\title{
Light Intensity Evaluation of Laser-induced Fluorescence after Caries Removal Using an Experimental Caries Staining Agent
}

\author{
Jun-Ichiro KINOSHITA, Hiroshi SHINOMIYA, Kazuo ITOH and Koukichi MATSUMOTO \\ Department of Clinical Cariology and Endodontology, Showa University School of Dentistry, 2-1-1 Kitasenzoku, Ohta-ku, \\ Tokyo 145-8515, Japan \\ Corresponding author, Jun-Ichiro KINOSHITA; E-mail: juun163@aol.com
}

Received October 19, 2006 /Accepted December 19, 2006

\begin{abstract}
Objective: The purpose of this study was to evaluate the intensity of fluorescent light reflected from cavity wall dentin, after dentin stained by an experimental caries detecting liquid was removed. Background Data: The conventional caries detecting liquid tends to penetrate dentin too deeply. On the other hand, DIAGNOdent ${ }^{\circledR}$ has gradually gained recognition and established itself as a caries detection device using a laser beam. Method: An experimental caries staining liquid or the conventional caries staining liquid was applied in the same cavity to observe for differences in dye penetration. Intensity of fluorescence reflected from treated dentin was measured by DIAGNOdent ${ }^{\circledR}$. Results: The mean DIAGNOdent ${ }^{\circledR}$ value after removing the tooth substance stained by the experimental liquid was $17.9 \pm 4.1$, compared with $7.7 \pm 2.7$ by conventional one. Conclusion: Light intensity evaluation of laser-induced fluorescence suggested that the experimental caries detecting liquid did not penetrate the dentin which should be preserved, hence avoiding overtreatment.
\end{abstract}

Keywords: Experimental caries detector, Sclerotic dentin, DIAGNOdent ${ }^{\circledR}$

\section{INTRODUCTION}

The 'conventional' caries detecting liquid was first introduced by Fusayama and Terashima, which was composed of $1 \%$ by weight of basic fuchsin in propylene glycol and of which the molecular weight was approximately $76.1^{1)}$. In their study, this solution selectively stained the outer layer of carious dentin. This is the region that should be removed since it contains irreversibly damaged collagen fibers which are unable to undergo recalcification ${ }^{2,3)}$. Conversely, it is desirable to conserve the inner layer of carious dentin since this region has potential for recalcification with appropriate treatment $t^{4,5)}$. However, it has been reported that caries detection using the conventional caries detecting liquid has resulted in overtreatment ${ }^{6,7)}$. To overcome the aforementioned, inadvertent overtreatment, we conceived the idea of using a thicker caries detecting liquid - to the end that it would not penetrate the inner layer of carious dentin. In the course of bringing this idea to fruition, we found that when a caries detecting fluid with a molecular weight of 300 reached the outer layer, it did not penetrate the inner layer. Upon further verification and confirmation by SEM and Vicker's hardness test results, this idea was finally presented at a conference in $2004^{8)}$.

Laser-induced fluorescence on tooth surface has also been used experimentally for caries detection. Lasers of various wavelengths have been tested and their reproducibility assessed-11). Following the footsteps of these pioneering researches, DIAGNOdent ${ }^{\circledR}(12 \times 15 \times 9 \mathrm{~cm})$ was introduced in 1999. It is a laser-based caries detection device which uses a semiconductor laser of less than $1 \mathrm{~mW}$ output power. It emits a beam of $655 \mathrm{~nm}$ wavelength from the tip of the laser handpiece, whereby fluorescence reflected from the tooth surface will be captured at the same tip. The score ranges from 00 to 99 - the smaller the healthier, and diagnosis can be performed immediately without damage to the tooth and with minimally invasive treatment to the patient. Accuracy of this device has been compared with that of radiography ${ }^{12}$, and its measurement results on occlusal and approximal caries have been shown to be acceptable ${ }^{13,14}$.

DIAGNOdent ${ }^{\circledR}$ has been reported to be useful not only in locating initial caries, but also in assessing the need for residual carious dentin removal. The score (e.g., D-20 or D-21) depends on the amount of metabolic by-products of cariescausing bacteria and fluorescent protoporphyrin present ${ }^{15)}$, and the color of carious dentin ${ }^{16)}$. In view of this diversified range of determining factors on carious dentin removal, some researchers have tried to establish a reliable set of excavation criteria for DIAGNOdent ${ }^{\circledR 17-19}$. Eventually, the criteria for caries detection and subsequent treatment options suggested by Lussi et al. are considered as the gold standard $^{20)}$. Based on their criteria, scores below D-13 would indicate that no active treatment is required; scores between D-14 to 20 would indicate preventive care and observation is required, since recalcification is feasible; scores between D-21 to 29 would indicate that preventive and operative care is required; and scores over D-29 indicate that caries removal is required.

At this juncture, it should be highlighted that some researchers expressed the view that 
DIAGNOdent ${ }^{\circledR}$ measurement was not so dependable because of its low rate of reproducibility in their experiments $^{21-23)}$. To these feedback comments, we pointed out the limited nature of the recommended technique by the product maker ${ }^{24)}$. We then introduced an improved measurement technique by demonstrating the results of an experiment using a video tape, which showed a much better reproducibility rate than the former technique ${ }^{24)}$. Essentially, the main concept behind our technique was to eliminate unnecessary accumulation of photon reflected from the surrounding surface of an examined point. As such, we proposed that different measurement techniques should be employed for clinical use and research use. This is because the purpose of the latter is not to determine the degree of caries severity in the lesion area. Our proposed technique was based on the supposition that this device could detect the discrepancy in photon amount between two closely neighboring points on a surface. It was reported that the average free path of a light photon was of the order of $0.5 \mathrm{~mm}$ in healthy tooth substance, while it traveled sideward for about 0.1 $\mathrm{mm}$ in carious tooth substance ${ }^{25}$ 26). In light of these data, we adopted the $0.5-\mathrm{mm}$ sideward movement in our new measurement method.

The purpose of the present study was to confirm that our experimental caries detecting liquid did not dye the dentin which has potential to be recalcified. To this end, an optical examination using DIAGNOdent ${ }^{\circledR}$ was carried out. By evaluating the light intensity of fluorescence reflected from treated dentin surface, it could serve to validate or disprove the potential use of this experimental caries detector.

\section{MATERIALS AND METHODS}

\section{Experimental caries detecting liquid}

For the experimental caries detecting liquid, it was composed of polypropylene glycol (with an average molecular weight of 300) and a red dye (Fig. 1). The preparation procedure for this experimental caries detecting liquid (as described below) was carried out with the assistance of a chemical company (Nippon Shika Yakuhin, Shimonoseki, Japan).

Triol-type polypropylene glycol, with an average molecular weight of 300 (Wako Junyaku Kogyo, Osaka), and Food Red No. 106 (Tokyo Kasei Kogyo, Tokyo) were stirred using an electric stirrer in a ratio of $99 \%$ by weight to $1 \%$ respectively. The mixture was passed through a filter to eliminate dust. With this formulation, the coefficient of viscosity was almost the same as that of the conventional caries detector.

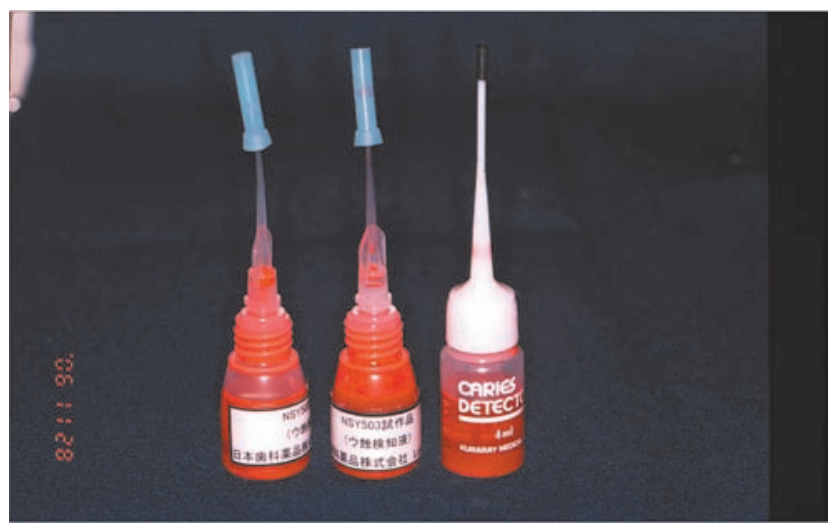

Fig. 1 Experimental caries detecting liquid (two leftmost bottles), and Caries Detector, the conventional caries detecting agent (rightmost bottle).

Sample selection and assessment by semiconductor laser device

Fifty-two freshly extracted human teeth with dentin caries (degree C2, not reaching pulp chamber, including both acute and chronic caries) were used in this study. Written informed consent was obtained from each individual for use of his/her extracted tooth. Tooth samples were kept in saline to minimize chemical artifacts manifested in fluorescence reflected from tooth substance, as well as to avoid crack formation on surface.

Caries lesions at various locations, of different depths and colors, were chosen from the 52 tooth samples. In each case, one drop of the experimental caries liquid was applied to the cavity for 10 seconds, rinsed off with a water spray attached to a treatment chair for five seconds, and the cavity dried by an air spray. Stained dentin was then completely removed using a round steel bur (ISO No. 6, diameter: $2 \mathrm{~mm})$ in a slow-speed handpiece $(100,000$ rpm), with water spray as coolant.

After removing the stained tooth substance, DIAGNOdent ${ }^{\circledR}$ (KaVo Dental GmbH, Jena, Germany) (Fig. 2) was used to evaluate the level of residual caries. The mean of five measurements was recorded as its D-score. The staining and cutting of dentin, as well as DIAGNOdent ${ }^{\circledR}$ measurement, were repeated until the cavity wall was no longer stained. After assuming complete removal of carious dentin by the experimental detecting liquid, a commercially available caries detecting liquid (Caries Detector, Kuraray, Okayama, Japan) was applied in the same cavity. Newly stained dentin was removed in the same way. The D-score obtained using the conventional caries detector was likewise recorded.

DIAGNOdent ${ }^{\circledR}$ measurements were carried out using the improved technique introduced in our previous report ${ }^{24}$. A tapered fiber optic tip (Tip A) of the handpiece was slowly lowered, as vertically as possible, to a location $0.5 \mathrm{~mm}$ over the red light- 


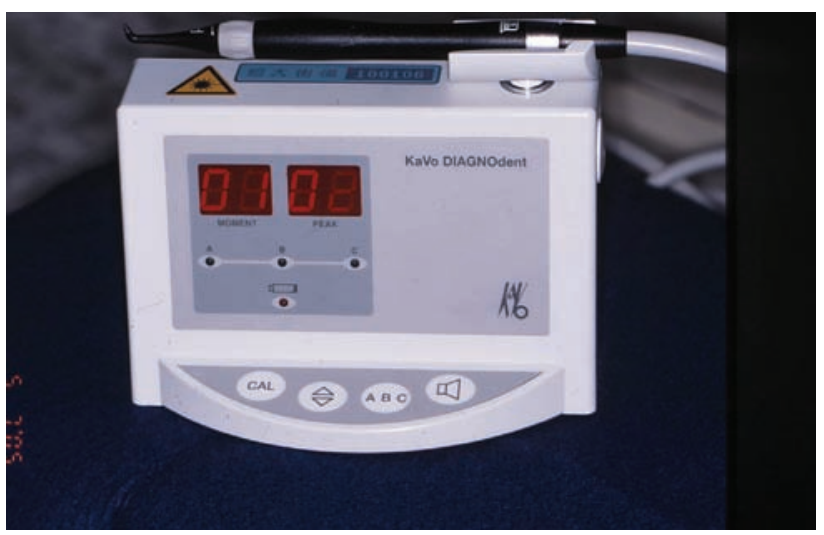

Fig. 2 Caries detection laser device, DIAGNOdent ${ }^{\circledR}$, with $655 \mathrm{~nm}$ semiconductor laser.

Table 1 D-scores after removing stained dentin

\begin{tabular}{lrl}
\hline & D-Score & P \\
\hline Experimental Caries Detector & $17.9 \pm 4.1(\mathrm{n}=30)$ & \\
Commercial Caries Detector & $7.7 \pm 2.7(\mathrm{n}=30)$ & $<0.005$ \\
Sound dentin & $5.3 \pm 0.6(\mathrm{n}=30)$ & \\
\hline
\end{tabular}

D-score values are represented as mean \pm SD of 52 teeth. Number of parentheses represents the number of measurement points.

$\mathrm{P}$ value was calculated by Kruskal-Wallis one-way analysis of variance by ranks.

irradiated surface. Then, it was quickly moved to right or left side by about $0.5 \mathrm{~mm}$. After which, it was instantly withdrawn upward from the irradiated surface. The maximum of digitally displayed numbers was recorded as the D-score for the examined point. This improved technique was different in that the tip was neither tilted nor moved on the lesion surface for a few seconds.

\section{Statistical analysis}

The mean D-score of sound human dentin based on measurements of 13 caries-free human teeth was used as a control. These three groups of D-scores were analyzed statistically using Kruskal-Wallis one-way analysis.

\section{RESULTS}

After removing the dentin stained by the experimental caries detector, all the cavity walls could still be stained by the conventional caries detector (Fig. 3). In other words, the dye of the conventional one would penetrate deeper into all the samples.

The D-score of sound dentin was 5.3 0.6. As for carious dentin treated with experimental liquid, the D-score was $17.9 \pm 4.1$, whereas that of carious dentin treated with conventional liquid was $7.7 \pm 2.7$
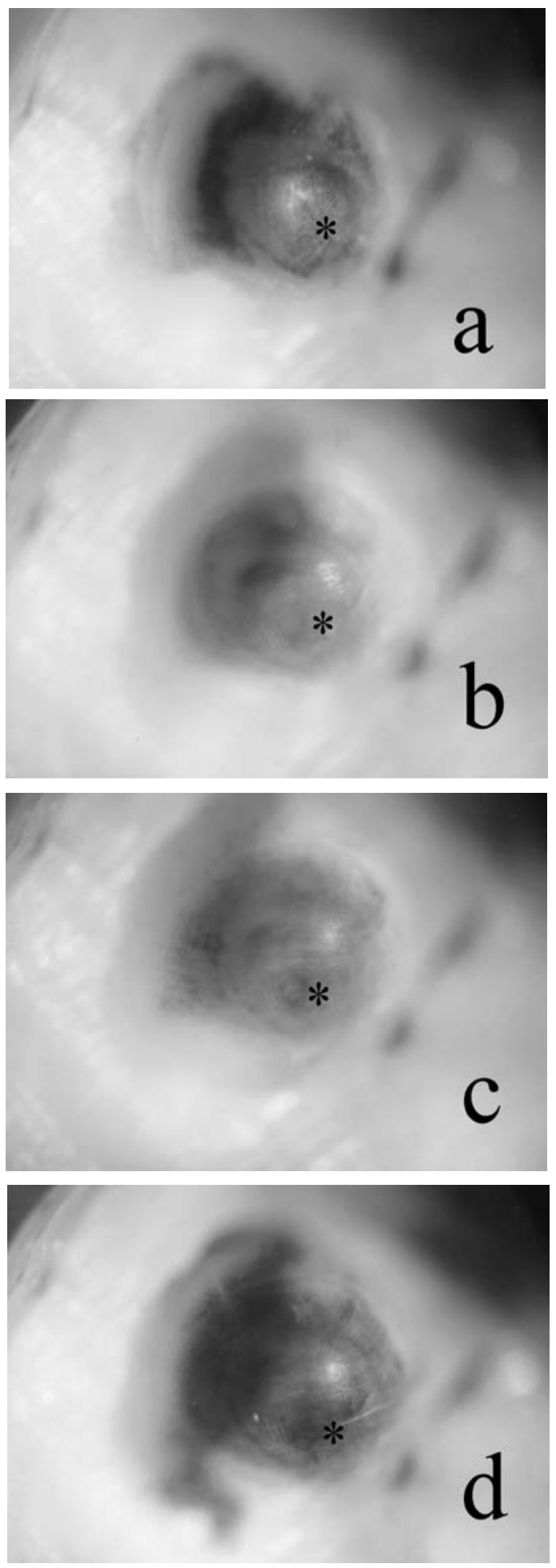

Fig. 3 (a) Cavity wall on tooth stained by the experimental caries detecting liquid; (b) Stained dentin by experimental liquid has been removed; (c) No further staining of cavity wall by experimental liquid is visible; (d) Conventional caries detecting liquid nonetheless stained it. (*: a mark to show the fixed point at which DIAGNOdent ${ }^{\circledR}$ measurement was done.) 
(Table 1). Based on Kruskal-Wallis one-way analysis of variance, there were significant differences among the three groups $(p<0.005)$.

\section{DISCUSSION}

Some researchers have reported that the conventional caries detecting liquid leads to excessive treatment $t^{27}$. This can be explained by the propensity of this type of dye agent to dye demineralized collagen and organic matrices rather than bacteria ${ }^{28)}$. Another researcher showed that within the carious dentin zone, a part of which was called sclerotic dentin, which was a little porous but uninfected ${ }^{29}$. This would mean that this inner layer of carious dentin is easily dyed by an agent of low molecular weight, although this tooth substance can be conserved when minimal intervention is intended.

With regard to dentin bonding, some researchers insisted that the presence of sclerotic dentin resulted in reduced bond strength ${ }^{30-32)}$. However, we found that especially in sclerotic dentin, priming with 35 vol\% glyceryl monomethacrylate solution decreased the width of contraction gaps, if not even completely prevented the formation of contraction gaps ${ }^{33}$. In light of this finding, the sclerotic dentin layer in inner carious dentin should be conserved not only for minimal intervention, but also for better adhesion ${ }^{34)}$.

With reference to the excavation criteria currently supported and endorsed by the majority of DIAGNOdent ${ }^{\circledR}$ researchers, D-scores below 13 indicate intact, healthy dentin or enamel; D-scores 14 to 20 indicate pseudo-carious dentin which can be recalcified; D-scores 21 to 29 indicate carious dentin which has no possibility to be recalcified; and Dscores above 29 to 99 indicate severely infected dentin. In the current study, the D-score after removing dentin stained by the conventional caries detecting liquid was D-7.7, suggesting that very sound dentin was exposed in cavity wall. In other words, sclerotic dentin had been completely removed. Such overcutting of dentin in this group was caused by the high penetration of the dye. In contrast, the D-score after removing dentin stained by the experimental caries detecting liquid was D-17.9, suggesting that this surface was composed of some intermediate substance between carious dentin and intact dentin. Based on DIAGNOdent ${ }^{\circledR}$ criteria, such a D-score was amenable to recalcification. Thus, it would not be irrational to suppose that this surface may exist in sclerotic dentin or pseudo-carious dentin.

Based on the D-score results obtained in this study, it could be said that by means of a caries detector with a higher molecular weight, even more improved minimal intervention approaches for managing dental caries could now be realized.

\section{CONCLUSION}

The new, experimental caries detecting agent was composed of polypropylene glycol with an average molecular weight of 300 - whereby this molecular weight was much higher than that of the conventional caries detector.

An optical evaluation of fluorescent light reflected from treated dentin surface confirmed that this new caries detecting agent might be useful in conserving dentin that can be recalcified. On the other hand, the conventional caries detector tended to result in overcutting of intact, healthy cavity wall dentin.

\section{ACKNOWLEDGEMENTS}

1. This investigation was supported by "HighTech Research Center" Project for Private Universities, with a matching fund subsidy from MEXT (Ministry of Education, Culture, Sports, Science and Technology), 2005-2008.

2. Immense gratitude is hereby rendered to Dr. Masayoshi Tunekawa, Director, R\&D, and his colleagues in Nippon Shika Yakuhin (Shimonoseki, Japan), for their encouragement toward the publication of this paper.

\section{REFERENCES}

1) Fusayama T, Terashima S. Differentiation of two layers of carious dentin by staining. Bull Tokyo Med Dent Univ 1972; 19:83-92.

2) Ohgushi K. Collagen fibers in the two layers of carious dentin. 1. Histochemical study. J Jpn Stomatol Soc 1973; 40:65-74.

3) Ohgushi K. Collagen fibers in the two layers of carious dentin. 2. Electron microscopic study. J Jpn Stomatol Soc 1973; 40:306-315.

4) Miyauchi H, Iwaku M, Fusayama T. Physiological recalcification of carious dentin. Bull Tokyo Med Dent Univ 1978; 25:169-179.

5) Fusayama T. Two layer of carious dentin: diagnosis and treatment. Oper Dent 1979; 4:63-70.

6) Fukushima M. Adhesive resin penetration into carious dentin. J Jpn Stomatol Soc 1981; 48:362-385.

7) Sano H. Relationship between caries detector staining and structural characteristics of carious dentin. J Jpn Stomatol Soc 1987; 48:241-270.

8) Oikawa M, Kusunoki K, Itoh K, Hisamitsu H. The experimental caries detector containing polypropylene glycol. J Dent Res 2004; 83: A-0757.

9) Angmar-Månsson B, ten Bosch JJ. Optical methods for the detection and quantification of caries. Adv Dent Res 1987; 1:14-20.

10) Hafström-Björkman U, Sundström F, de Josselin de Jong E, Oliveby A, Angmar-Månsson B. Comparison of laser fluorescence and longitudinal microradiography for quantitative assessment of in vitro enamel caries. Caries Res 1992; 26:241-247.

11) Barron J, Paton B, Zakariasen K. Micro-analysis of 
dental caries using laser scanned fluorescence. SPIE 1992; Vol. 1643 Laser Surgery: 503-509.

12) Shi $X Q$, Welander U, Angmar-Månsson B. Occlusal caries detection with KaVo DIAGNOdent and radiography: an in vitro comparison. Caries Res 2000; 34:151-158.

13) Lussi A, Imwinkelried S, Pitts NB, Longbottom C, Reich E. Performance and reproducibility of a laser fluorescence system for detection of occlusal caries in vitro. Caries Res 1999; 33:261-266.

14) Lussi A, Hack A, Hug I, Heckenberger H, Megert B, Stich H. Detection of approximal caries with a new laser fluorescence device. Caries Res 2006; 40:97-103.

15) König K, Flemming G, Hibst R. Laser-induced autofluorescence spectroscopy of dental caries. Cell Mol Biol 1998; 44:1293-1300.

16) Iwami Y, Shimizu A, Hayashi M, Takeshige F, Ebisu S. Relationship between colors of carious dentin and laser fluorescence evaluations in caries diagnosis. Dent Mater J 2006; 25:584-590.

17) Shinohara T, Takase Y, Hirai Y. Criteria for the clinical diagnosis of caries through the application of laser. Japan J Conserv Dent 2004; 47:22-32.

18) Kinoshita J, Kimura Y, Matsumoto K. Comparative study of carious dentin removal by Er, Cr: YSGG laser and Carisolv. J Clin Laser Med Surg 2003; 21:307-315

19) Shinohara $T$, Takase $Y$, Amagai $T$, Haruyama $C$, Igarashi A, Kukidome N, Kato J, Hirai Y. Criteria for a diagnosis of caries through the DIAGNOdent. Photomed Laser Surg 2006; 24:50-58.

20) Lussi A, Hibst R, Paulus R. DIAGNOdent: An optical method for caries detection. J Dent Res 2004; 83:C80-C83.

21) Baseren NM, Gokalp S. Validity of a laser fluorescence system (DIAGNOdent) for detection of occlusal caries in third molars: an in vitro study. J Oral Rehabil 2003; 30:1190-1194.

22) Kuhnisch J, Ziehe A, Brandstadt A, Heinrich-Weltzien $\mathrm{R}$. An in vitro study of the reliability of DIAGNO dent measurements. J Oral Rehabil 2004; 31:895-899.
23) Bader JD, Shugars DA. A systematic review of the performance of a laser fluorescence device for detecting caries. JADA 2004; 135:1413-1426.

24) Kinoshita JI, Kimura Y, Nagai K, et al. Measurement of infected root canal dentin chips by DIAGNOdent $^{\mathrm{rm}}$. Japan J Conserv Dent 2005; 48:362371.

25) Spitzer D, ten Bosh JJ. The absorption and scattering of light in bovine and human dental enamel. Calcif Tissue Res 1975; 17:129-137.

26) ten Bosh JJ, van der Mei HC, Borsboom PCF. Optical monitor of in vitro caries. A comparison with chemical and microradiographical determination of mineral loss in early lesions. Caries Res 1984; 18:540-547.

27) Yip HK, Stevenson AG, Beeley JA. The specificity of caries detector dyes in cavity preparation. Br Dent $\mathrm{J}$ 1994; 176:417-421.

28) Boston DW, Graver HT. Histological study of an acid red caries-disclosing dye. Oper Dent 1989; 14:186192.

29) Banerjee A, Kidd EA, Watson TF. In vitro validation of carious dentin removed using different excavation criteria. Am J Dent 2003; 16:228-230.

30) Nakajima M, Sano H, Burrow MF, et al. Tensile bond strength and SEM evaluation of caries-affected dentin using dentin adhesives. J Dent Res 1995; 74:1679-1688.

31) Yoshiyama M, Sano H, Ebisu S, et al. Regional strength of bonding agents to cervical sclerotic root dentin. J Dent Res 1996; 75:1404-1413.

32) Yoshiyama M, Carvalho RM, Sano H, Horner JA, Brewer PD, Parshley DH. Regional bond strengths of resins to human root dentine. J Dent 1996; 24:435442.

33) Tani C, Itoh K, Hisamitsu H, Wakumoto S. Efficacy of dentin bonding to cervical defects. Dent Mater $J$ 2001; 20:359-368.

34) Kusunoki M, Itoh $\mathrm{K}$, Takahashi $\mathrm{Y}$, Hisamitsu H. Contraction gap versus shear bond strength of dentin adhesive in sound and sclerotic dentins. Dent Mater J 2006; 25:576-583. 